\title{
Comparing Height-Adjusted Waist Circumference Indices: The Fels Longitudinal Study
}

\author{
Roy T. Sabo, Chungfeng Ren, Shumei S. Sun \\ Department of Biostatistics, School of Medicine, Virginia Commonwealth University, Richmond, USA \\ Email: rsabo@vcu.edu
}

Received May 11, 2012; revised June 20, 2012; accepted July 12, 2012

\begin{abstract}
Objectives: While researchers are increasingly recognizing the importance of adjusting waist circumference (WC) for height, no standard has yet been established. In this study we contrast three standard methods for indexing WC by height (using height, root-height and height-squared) via comparisons with age-specific optimal indices. Study Design and Setting: Measurements from 722 male and 746 female Caucasian participants in the Fels Longitudinal Study were used. The three standard waist-circumference indices (as well as an optimal index) were determined for ages 2 through 18, and for every decade thereafter to 70 years of age. Pearson correlations were used to assess the suitability of all indices. Results: The three standard indices remain correlated with the original WC measures, though each was associated with height at some ages. Waist-to-height ratio is suitable for some childhood ages (boys: 5 - 9, 13 - 16; girls: 4 - 7, 9, 11 - 14) but not for adult ages; Root-height works well mostly for older teenage children and adults but not in early childhood and adolescence; Height-squared is nowhere suitable. In both men and women, the optimal indexing factor ranged between root-height and height-squared in childhood, and is close to root-height in adulthood. Conclusions: No one index is most suitable, as WC indexed by root-height is suitable for use with measurements from teenage children and adults, while waist-to-height ratio is generally suitable for use in children. WC indexed by height-squared is nowhere suitable.
\end{abstract}

Keywords: Obesity; Adiposity; Stature; Indexing

\section{Introduction}

Several body composition and cardiac structure and function measurements experience improved predictability of obesity and cardiovascular health measures when they are indexed by height (or a power of height), including body mass index (BMI) [1] and left ventricular mass [2,3]. Waist circumference (WC) is an adiposity measure that, if properly adjusted by some power of height [2], can add predictive ability of childhood body weight to adult blood pressure [4,5]. Several recent studies have found that waist-to-height ratio (WHtR) is better than WC at predicting obesity, overweight [6], cardiovascular risk factors [7] and mortality [8], is better than BMI at predicting hypertension, diabetes, dyslipidemia [9], metabolic syndrome [10] and its risk factors [11], type II diabetes [12] and cardiovascular risk factors [7], and is better than waist-to-hip ratio (WHR) at predicting cardiovascular risk factors [7] and mortality[8]. WHtR also has the added benefit of not requiring adjustment for age or sex [13], though that may still be done. Some studies have shown no difference between WHtR, WC and BMI in predicting cardiovascular risk factors [14], while others have shown that WHtR is inferior to WC as an index of trunk fat mass [15].

Implicit in these studies and their use of WHtR is the choice of indexing waist circumference by a subject's height raised to the first power. Though the reason for indexing WC by height is obvious, and the resulting fidelity of such an index is clear, the rationale for indexing by subject height and not some other power of height is less well developed. For instance, the square of height could be used so that the resulting WC index would parallel the index for body mass (BMI = body mass/height ${ }^{2}$ ). The square-root of height could also be used to index WC, as could any other radical number between zero and one. The problem of properly indexing WC by height is not merely a problem of choice, however, as the exponent for height in any index should be selected so that something is optimized. For instance, Khosla and Lowe (1967) found that 2.0 was the optimal exponent for indexing body mass by height in adults because that particular exponent generated an index that was highly correlated with body mass and was uncorrelated with height [1]. Likewise, an exponent for height should be found so that the resulting index of WC would be highly correlated with waist circumference and uncorrelated 
with height.

The purpose of this study is to compare three commonly methods used for indexing waist circumference by height (waist-to-height ratio, using the square root of height, and using height-squared) in male and female children and adults. Using data from the Fels Longitudinal Study, the three waist circumference indices-as well as the optimal powers for indexing waist circumference by height-are estimated for male and female children at each age between 2 and 18 years of age, and for male and female adults at ten-year intervals from the third through eighth decades. Correlative analyses are then used to determine 1) Whether each index remains correlated with the original waist circumference measures not indexed by height; and 2) Whether each index is uncorrelated with the original height measure (i.e. has the index "factored out" the effect of height from waist circumference).

\section{Methods}

\subsection{Study Sample}

In this study we analyzed anthropometric data on 722 Caucasian male and 746 Caucasian female participants of the Fels Longitudinal Study (FLS) between 2 and 70+ years of age as of 6/30/2010. FLS participants were enrolled at birth and were not selected in regard to factors known to be associated with disease, body composition or other conditions [16]. Each participant signed an informed consent statement, and procedures were approved by the Institutional Review Boards at Wright State University and Virginia Commonwealth University.

\subsection{Measurements}

Anthropometric measurements were taken following recommendations in the Anthropometric Standardization Reference Manual [17]. Waist circumference (WC, cm) was measured twice at the suprailiac crest, with the average value used for statistical analysis. Height was measured to $0.1 \mathrm{~cm}$ using a Holtain stadiometer. Children were examined semi-annually (near their birthdays and 6 months following) until 18 years of age, then biannually to 24 years of age. Subsequent adult measurements occurred at fiveyear intervals until age 40; thereafter, measurements were taken at two-year intervals.

\subsection{Statistical Analyses}

We summarize waist circumference and height for each gender at every childhood age and adult decade with means and standard deviations. Waist-to-height ratio (WHtR), WC indexed by the square root of height (WCI1), and WC indexed by height-squared (WCI2) are calculated for each subject at each available age. These indices will be graphically represented with means and standard errors for both genders at each age between 2 and 18 and at each decade thereafter. As an attempt to verify the suitability of three WC indices (as well as the optimal index (aWCI), explained below) for adequately measuring WC without being affected by subject height, we will follow the approach used by Khosla and Lowe (1967), who stated that an adequate index (i) should be highly correlated with the metric upon which it is based (in this case WC), and (ii) should be uncorrelated with height. We will estimate Pearson correlations to measure the associations of WHtR, WCI1, WCI2 and aWCI with both WC and height at each age in each gender. The SAS statistical software (version 9.2, Cary, North Carolina, USA) is used for all statistical analyses, and a significance level of $\alpha=0.05$ is used for all tests.

To determine the optimal power for height in indexing waist circumference, we follow the method used by Khosla and Lowe (1967), who concluded that the square of height was optimal to index body mass in adults [1]. The predicted value of waist circumference ( $\widehat{\mathrm{WC}}$ ) is given by the equation.

$$
\widehat{\mathrm{WC}}=\overline{\mathrm{WC}}+\beta(H-\bar{H})
$$

where $\overline{\mathrm{WC}}$ is the mean waist circumference, $\beta$ is the regression slope between height and waist circumference, and $H$-bar is the mean height. We then define

$H_{b}=\bar{H}+3 \times S D$ and $H_{a}=\bar{H}-3 \times S D$, such that

$\widehat{\mathrm{WC}}_{b}=\overline{\mathrm{WC}}+\beta\left(H_{b}-\bar{H}\right)$ and

$\widehat{\mathrm{WC}}_{a}=\overline{\mathrm{WC}}+\beta\left(H_{a}-\bar{H}\right)$, and the optimal exponent is calculated using the following equation:

$$
e=\frac{\log \left(\widehat{\mathrm{WC}}_{b}\right)-\log \left(\widehat{\mathrm{WC}}_{a}\right)}{\log \left(H_{b}\right)-\log \left(H_{a}\right)}
$$

We calculate this exponent each age between 2 and 18 years separately for boys and girls, and at 10 year intervals thereafter (20 - 29, 30 - 29, 40 - 49, 50 - 59, 60 - 69 and $70+$ ) for men and women, where any repeated measures for a given subject within any given interval are averaged together. These powers are then used to calculate age-specific indices for waist circumference (aWCI).

\section{Results}

The data for waist circumference (WC) and height are summarized for boys and adult men in Table $\mathbf{1}$ and for girls and adult women in Table 2. Note that means of both WC and height take similar values for both boys and girls until around age 14, at which point boys start to increase in height more than girls, though there is not much of a gender discrepancy for WC. Based on the adult age-intervals, males generally have greater WC and height than women throughout adulthood. Note also that 
Table 1. Male summary data. Sample sizes (N), mean waist circumference (WC) and standard deviation (SD), mean height and standard deviation, regression slope $(\beta)$ between weight and centered height, and age-specific optimal power for boys aged 2 to 18 years and adult men in ten-year intervals between ages 20 to $70+$.

\begin{tabular}{cccccccc}
\hline \multicolumn{2}{c}{ WC } & \multicolumn{2}{c}{ Height } & & \\
\hline Age & N & Mean & SD & Mean & SD & $\beta$ & Power \\
\hline 2 & 51 & 48.61 & 2.77 & 88.22 & 3.75 & 0.29 & 0.52 \\
3 & 125 & 50.14 & 3.01 & 94.97 & 4.05 & 0.29 & 0.56 \\
4 & 144 & 51.71 & 3.15 & 102.4 & 4.23 & 0.29 & 0.58 \\
5 & 164 & 53.48 & 4.34 & 108.7 & 4.67 & 0.39 & 0.80 \\
6 & 173 & 55.29 & 5.32 & 116.0 & 4.71 & 0.54 & 1.13 \\
7 & 178 & 57.14 & 6.67 & 122.9 & 6.30 & 0.58 & 1.24 \\
8 & 179 & 58.71 & 7.19 & 128.7 & 5.34 & 0.63 & 1.38 \\
9 & 169 & 61.22 & 8.27 & 134.4 & 5.61 & 0.63 & 1.38 \\
10 & 183 & 64.54 & 9.98 & 140.5 & 5.98 & 0.82 & 1.81 \\
11 & 192 & 66.34 & 10.29 & 145.5 & 6.15 & 0.82 & 1.81 \\
12 & 195 & 69.15 & 10.92 & 151.2 & 6.73 & 0.75 & 1.67 \\
13 & 197 & 71.48 & 10.76 & 157.8 & 8.14 & 0.60 & 1.34 \\
14 & 197 & 74.67 & 11.70 & 166.1 & 7.92 & 0.47 & 1.06 \\
15 & 188 & 76.91 & 12.31 & 171.6 & 7.37 & 0.50 & 1.12 \\
16 & 186 & 77.93 & 11.41 & 175.5 & 6.93 & 0.41 & 0.93 \\
17 & 190 & 80.35 & 11.41 & 177.9 & 6.56 & 0.16 & 0.35 \\
18 & 176 & 81.04 & 10.61 & 178.9 & 6.86 & 0.17 & 0.36 \\
$20-29$ & 265 & 87.43 & 12.99 & 180.1 & 7.10 & 0.24 & 0.50 \\
$30-39$ & 235 & 95.36 & 13.47 & 180.0 & 7.46 & 0.36 & 0.68 \\
$40-49$ & 230 & 98.72 & 11.73 & 179.6 & 7.24 & 0.20 & 0.36 \\
$50-59$ & 181 & 103.8 & 12.27 & 179.1 & 7.30 & 0.22 & 0.38 \\
$60-69$ & 100 & 109.0 & 12.92 & 177.6 & 7.17 & 0.05 & 0.08 \\
$70+$ & 61 & 107.6 & 11.34 & 174.3 & 6.13 & 0.34 & 0.55 \\
\hline & & & & & & &
\end{tabular}

Table 2. Female summary data. Sample sizes (N), mean waist circumference (WC) and standard deviation (SD), mean height and standard deviation, regression slope $(\beta)$ between weight and centered height, and age-specific optimal power for girls aged 2 to 18 years and adult women in ten-year intervals between ages 20 to $70+$.

\begin{tabular}{cccccccc}
\hline & \multicolumn{2}{c}{ WC } & \multicolumn{2}{c}{ Height } & & \\
\hline Age & N & Mean & SD & Mean & SD & $\beta$ & Power \\
\hline 2 & 68 & 48.59 & 2.96 & 87.86 & 3.60 & 0.38 & 0.68 \\
3 & 146 & 49.50 & 2.74 & 94.31 & 3.87 & 0.43 & 0.82 \\
4 & 165 & 51.34 & 3.36 & 101.4 & 4.34 & 0.46 & 0.91 \\
5 & 155 & 52.62 & 3.84 & 107.7 & 4.77 & 0.46 & 0.95 \\
6 & 170 & 54.66 & 4.85 & 115.3 & 5.75 & 0.56 & 1.19 \\
7 & 165 & 56.84 & 5.63 & 121.5 & 5.57 & 0.58 & 1.24 \\
8 & 173 & 59.23 & 6.30 & 128.2 & 5.93 & 0.61 & 1.32 \\
9 & 166 & 62.34 & 7.49 & 134.1 & 6.26 & 0.56 & 1.20 \\
10 & 169 & 64.93 & 8.55 & 140.3 & 6.96 & 0.73 & 1.59 \\
11 & 172 & 66.91 & 8.40 & 146.6 & 7.26 & 0.56 & 1.23 \\
12 & 172 & 70.10 & 9.00 & 153.4 & 7.23 & 0.55 & 1.20 \\
13 & 179 & 72.35 & 8.48 & 158.7 & 6.85 & 0.49 & 1.08 \\
14 & 183 & 74.06 & 8.68 & 161.4 & 6.85 & 0.33 & 0.72 \\
15 & 160 & 76.01 & 8.76 & 163.9 & 6.65 & 0.16 & 0.35 \\
16 & 170 & 76.97 & 9.08 & 164.3 & 6.56 & 0.16 & 0.34 \\
17 & 167 & 78.24 & 9.61 & 164.7 & 6.45 & 0.17 & 0.35 \\
18 & 179 & 78.97 & 9.98 & 165.5 & 6.60 & 0.07 & 0.15 \\
$20-29$ & 260 & 82.46 & 11.28 & 165.4 & 6.46 & 0.19 & 0.38 \\
$30-39$ & 268 & 87.75 & 13.20 & 166.3 & 6.11 & 0.27 & 0.51 \\
$40-49$ & 265 & 91.08 & 15.03 & 165.9 & 5.79 & 0.22 & 0.40 \\
$50-59$ & 185 & 93.30 & 12.93 & 164.6 & 6.09 & 0.27 & 0.48 \\
$60-69$ & 120 & 95.14 & 11.68 & 163.9 & 6.13 & 0.18 & 0.32 \\
$70+$ & 73 & 95.01 & 11.29 & 160.4 & 6.88 & 0.20 & 0.34 \\
\hline & & & & & & &
\end{tabular}


not all subjects provided both WC and height measurements for all ages, resulting in lower age-specific sample sizes than the total number of subjects reported in the Methods Section.

Using the equation to calculate the optimal power for indexing WC by height given in the Methods Section, we see that the estimated powers (resulting from the corresponding regression coefficients $(\beta)$ between height and WC) increase and decrease in a similar fashion as the estimated slopes. For boys (Table 1), the estimated power is 0.52 at age 2, increasing until it reaches a maximum of 1.81 at ages 10 and 11 , then decreasing until it reaches 0.35 at age 17 . The optimal powers then range between 0.36 and 0.68 between ages 20 and 60. These values imply that roots of height are optimal for indexing WC from ages 2 through 5, powers greater than 1 (but less than 2) are optimal between ages 6 and 15, and roots are again optimal from ages 16 to 18 and then on into adulthood. A similar pattern is observed in girls (Table 2), where the estimated power starts at 0.68 for age 2, increases until reaching a maximum of 1.59 at age 10 , then decreases until reaching 0.15 at age 18 . The optimal powers then range from 0.32 to 0.51 between ages 20 and $70+$. These values imply that roots of height are optimal for indexing WC from ages 2 through 5, powers greater than 1 (and less than 2) are optimal from ages 6 through 13, and roots of height are optimal from ages 14 through 18 and then on into adulthood.

Figure 1 contains age-specific plots of the WC-index means in boys and adult men for each of the four indexing methods along with standard errors (though they are too small to be noticed). Notable among the trends is the pronounced "rebound" in aWCI near age 11, which is not present in the other three indices (WHtR, WCI1 and WCI2). Note that the "rebound" observed around age 18 in WCI2 is due to the scale-shift from the annual childhood measurements to the decade-long adulthood intervals. A similar pattern is observed in girls in Figure 2, though the "rebound" in aWCI occurs slightly earlier in girls than in boys. In the plots for both male and female subjects, the aWCI, WHtR and WCI1 means are everywhere larger than the WCI2 means, which is expected since indexing by height squared leads to a larger divisor than does the indexing for the other methods, where none of the exponents for height are as large as 2. For both boys and girls aged 2 through 9, the aWCI and WHtR means are close in value, whereas from 10 through 14, the aWCI means are less than the WHtR means (since the aWCI exponent is larger at these ages than the 1.0 used in WHtR), and after 16 and into adulthood, the aWCI means are greater than the WHtR means (since the aWCI exponents at these ages are smaller than 1.0). Likewise, the means for aWCI are smaller than the means for WCI1 from around age 5 to around age 15 due to the optimal exponents in aWCI being larger than the 0.5 used in WCI1.

Based on Pearson correlations (Tables 3 and 4), we see that all four of the indices remain significantly $(\mathrm{p}<$ 0.001 ) and positively associated with the original WC measurement at all ages in both genders. Thus, each of these indices still capture-to some extent-the bodysize measurement of the original WC. Generally speak-

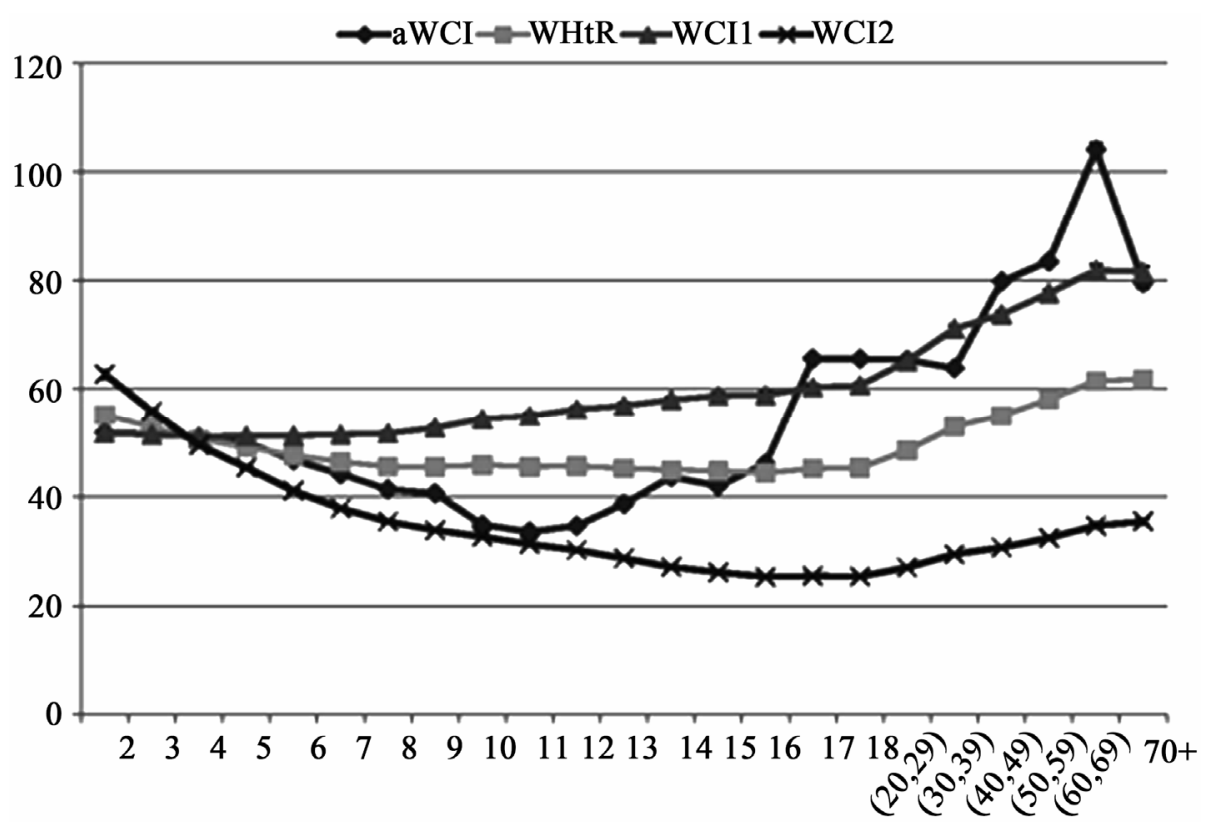

Figure 1. Male waist circumference indices. Means for waist circumference indices adjusted by: age-specific powers of height (aWCI), height (WHtR), the square root of age (WCI1), and height-squared (WCI2), for male subjects over ages 2 through 18, then by decade until 70+. 


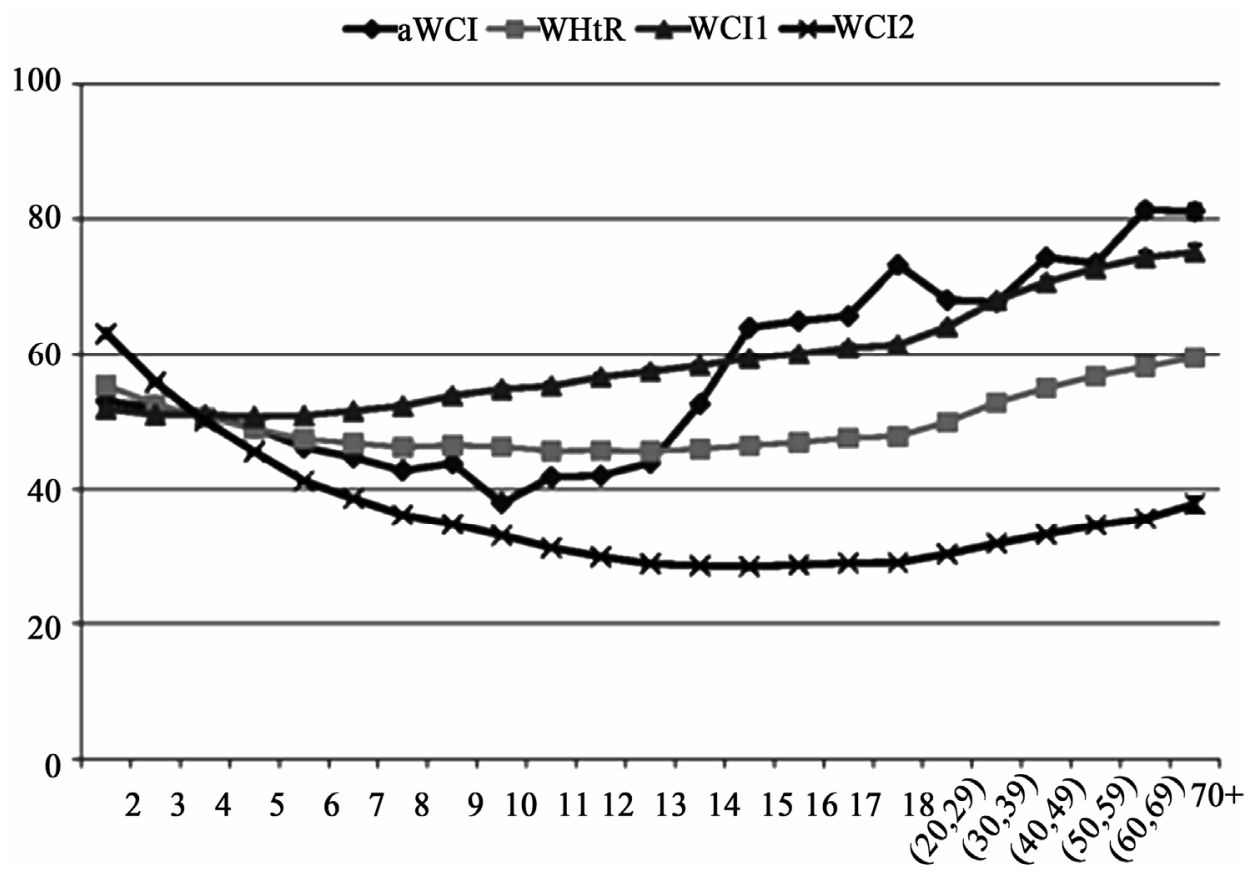

Figure 2. Female waist circumference indices. Means for waist circumference indices adjusted by: age-specific powers of height (aWCI), height (WHtR), the square root of age (WCI1), and height-squared (WCI2), for female subjects over ages 2 through 18, then by decade until $70+$.

ing, WCI1 (using root-height) is the most strongly associated index - of the four-with the original WC measurements in children and adults, with Pearson correlations everywhere between 0.93 and 0.99 for both males and females. WCI2 (using height-squared) is generally the least associated with the original WC measurements, with Pearson correlations ranging from 0.29 to 0.88 in males 0.10 to 0.91 in females. Both WHtR (waist-toheight ratio) and aWCI (the optimal index) are more strongly associated with the original WC measurements in latter adolescence and adult-hood than in childhood years.

Of the four indices, only aWCI was not associated with height at all ages and for both genders; this is expected since it is an "optimal index". WHtR is significantly and negatively associated with height in young children (ages 2 - 4 in boys; age 3 in girls), significantly and positively associated at some ages in adolescents (ages 10 12 in boys; age 10 in girls), and significantly and negatively associated with older teens and adults (ages 17 - 29, 40 - 69 in males; ages 15 - 49, 60+ in females). WCI1 is significantly and positively associated with height in young children and adolescents (ages 5 - 15 in booys; ages 3 - 13 in girls), yet is not associated with Height in adulthood for either gender. WCI2 is significantly and negatively associated with height throughout lifetimes in both males (ages 2 9, $13-70^{+}$) and females (ages $2-70^{+}$). The estimated correlations for WCI2-when significant-are generally larger than the estimated correlations for WHtR and WCI1.

\section{Discussion}

Just as body mass can be a misleading measure of the degree of obesity without indexing for height, waist circumference should also be indexed by some function of height in order for the measure to increase its utility as a body-size biomarker. Our analyses found that the performances of the three commonly used indices were age specific. Notably, WHtR had suitable properties for young boys and girls (roughly ages 2 - 15), while WCI1 (using root-height) was suitable for teenage and adult males and females (roughly ages $16-70+$ ). WCI2 (using height-squared) was nowhere suitable.

Our definition of "suitable" here mimics that used in a study for the proper indexing of body mass index with height by Kholsa and Lowe [1], where a desirable index would be correlated with the original body size measures (here WC) and would be uncorrelated with the indexing agent (here height). By repeating their methods for WC, we found the optimal exponents for indexing WC by height to be highly age-dependent (for both sexes) in childhood ranging from roots in early childhood, powers greater than one and less than two in adolescence, and then roots for latter teenage and adult ages. This explains why none of the commonly used WC indices work well for all ages, and especially explains why WC indexed by height-squared is nowhere suitable (the optimal values never reach two). Further studies are needed to discern whether a more complicated method for indexing WC by height is warranted. 


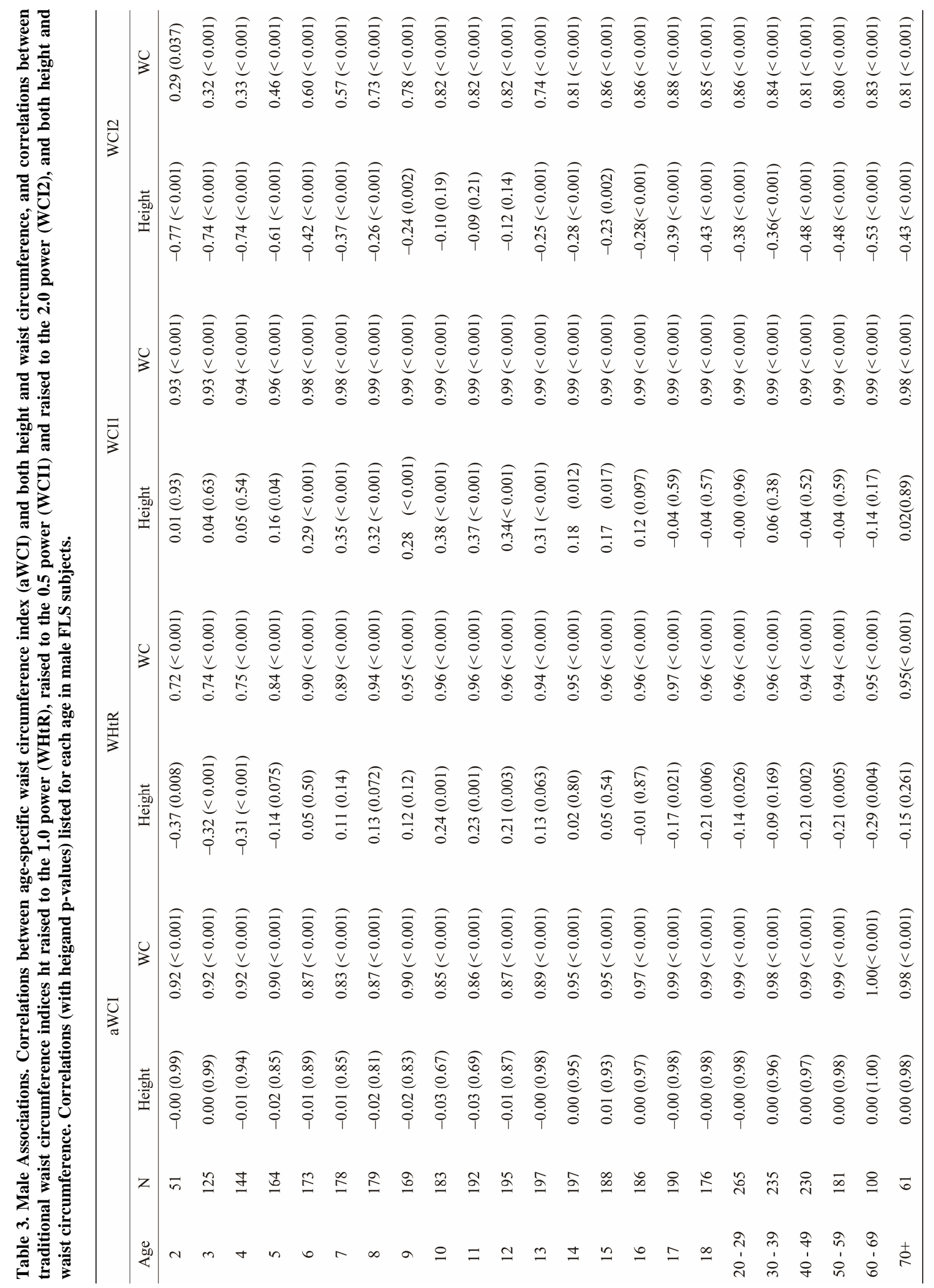




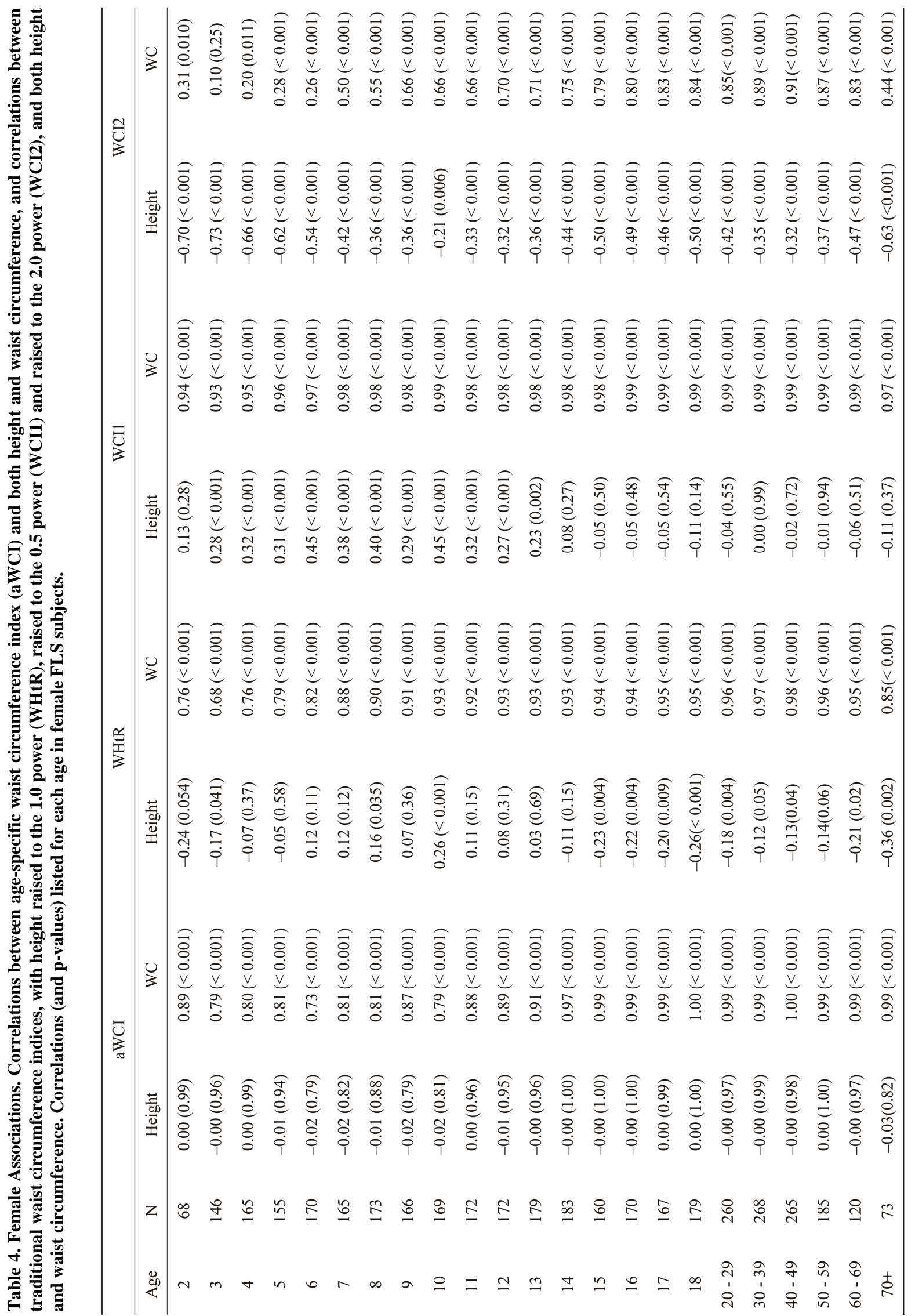


An important limitation of this study is that the Fels Longitudinal Study data base contains few non-white subjects; nearly all of the FLS subjects in this study are white. Thus, care should be used in interpreting the results from this study, and any interpolations to races other than Caucasian should be avoided.

Confirmation of these results in other cohorts should be considered. Another step in this research will be to investigate the predictive power of WHtR and WCI1 with respect to other obesity and cardiovascular health outcomes. Many studies have compared WHtR with other obesity measures, like BMI and WC, and used receiver-operating characteristic (ROC) curves on which to base the comparisons $[7,9,10,14,18]$. Fortunately, the FLS database contains many of the same obesity and cardiovascular health outcome measurements used in those studies, so that any new results can be compared with previous results.

\section{Acknowledgements}

The research which resulted in this manuscript was supported by grants U01HL101064-02, R01HD060913-03, R01HD038356, DK 071485, HL 072838 and HD 12252 from the National Institutes of Health, Bethesda, Maryland.

\section{REFERENCES}

[1] T. Khosla and C. Lowe, "Indices of Obesity Derived from Body Weight and Height,” British Journal of Preventive and Social Medicine, Vol. 21, No. 3, 1967, pp. 122-128.

[2] S. Daniels, R. Meyer, Y. Liang and K. Bove, "Echocardiographically Determined Left Ventricular Mass Index in Normal Children, Adolescents and Young Adults," The Journal of the American College of Cardiology, Vol. 12, No. 3, 1988, pp. 703-708.

[3] R. Lang, M. Bierig, R. Deverux, F. Flachskampf, E. Foster and P. Pellikka, "Recommendations for Chamber Quantificaiton: A Report from the American Society of Echocardioagraphy's Guidelines and Standard Committee and the Chamber Quantification Writing Group, Developed in Conjunction with the European Association of Echocardiography, a Branch of the European Society of Cardiology," Journal of the American Society of Echocardiography, Vol. 18, No. 12, 2005, pp. 1440-1463. doi:10.1016/j.echo.2005.10.005

[4] R. Lauer, T. Burns, W. Clarke and L. Mahoney, "Childhood Predictors of Future Blood Pressure,” Hypertension, Vol. 18, No. 3, 1991, pp. 174-181. doi:10.1161/01.HYP.18.3_Suppl.I74

[5] R. Gillum, "The Association of Body Fat Distribution with Hypertension, Hypertensive Heart Disease, Coronary Heart Disease, Diabetes and Cardiovascular Risk Factors in Men and Women Aged 18 - 79 Years,” Journal of Chronic Diseases, Vol. 40, No. 5, 1987, pp. 421-428. doi:10.1016/0021-9681(87)90175-5
[6] W. Yan, H. Bingxian, Y. Hua, D. Jianghong, C. Jun, G. Dongliang, Z. Yugian, L. Ling, G. Yanying, X. Kaiti, F. Xiaohai and M. Da, "Waist-to-Height Ratio Is an Accurate and Easier Index for Evaluating Obesity in Children and Adolescents,” Obesity, Vol. 15, No. 3, 2007, pp. 748752. doi:10.1038/oby.2007.601

[7] S.-Y. Ho, T.-H. Lam, E. Janus and H. K. C. R. F. P. S. S. Committee, "Waist to Stature Ratio Is More Strongly Associated with Cardiovascular Risk Factors than Other Simple Anthropometric Indices,” Annals of Epidemiology, Vol. 13, No. 10, 2003, pp. 683-691. doi:10.1016/S1047-2797(03)00067-X

[8] H. Schneider, N. Friedrich, J. Klotsche, L. Pieper, M. Nauck, U. John, M. Dorr, S. Felix, H. Lehnert, D. Pittrow, S. Silber, H. Volzke, G. Stalla, H. Wallaschofski and H. Wittchen, "The Predictive Value of Different Measures of Obesity for Incident Cardiovascular Events and Mortality," Journal of Clinical Endocrinology and Metabolism, Vol. 95, No. 4, 2010, pp. 1777-1785. doi:10.1210/jc.2009-1584

[9] C. Lee, R. Huxley, R. Wildman and M. Woodward, "Indices of Abdominal Obesity Are Better Discriminators of Cardiovascular Risk Factors than BMI: A Meta-Analysis,” Journal of Clinical Epidemiology, Vol. 61, No. 7, 2008, pp. 646-653. doi:10.1016/j.jclinepi.2007.08.012

[10] K. Knowles, L. Paiva, S. Sanchez, L. Revilla, T. Lopez, M. Yasuda, N. Yanez, B. Gelaye and M. Williams, "Waist Circumference, Body Mass Index, and Other Measures of Adiposity in Predicting Cardiovascular Disease Risk Factors among Peruvian Adults," International Journal of Hypertension, Vol. 2011, Article ID 931402, 10 pages.

[11] A. Bosy-Westphal, C. Geisler, S. Onur, O. Korth, O. Selberg, J. Schrezenmeir and M. Muller, "Value of Boy Fat Mass vs Anthropometric Obesity Indices in the Assessment of Metabolic Risk Factors," International Journal of Obesity, Vol. 30, 2005, pp. 475-483. doi:10.1038/sj.ijo.0803144

[12] F. Hadaegh, A. Zabetian, H. Harati and F. Azizi, "Waist/ Height Ratio as a Better Predictor of Type 2 Diabetes Compared to Body Mass Index in Tehranian Adult Men: A 3.6-Year Prospective Study," Experimental and Clinical Endocrinology \& Diabetes, Vol. 114, No. 6, 2006, pp. 310-315. doi:10.1055/s-2006-924123

[13] C. Maffeis, C. Banzato, G. Talamini and G. Talamini, "Waist-to-Height Ratio, a Useful Index to Indentify High Metabolic Risk in Overweight Children," The Journal of Pediatrics, Vol. 152, No. 2, 2008, pp. 207-213. doi:10.1016/j.jpeds.2007.09.021

[14] L. Paniagua, V. Lohsoonthorn, S. Lertmaharit, W. Jiamjarasrangsi and M. Williams, "Comparison of Waist Circumference, Body Mass Index, Percent Body Fat and Other Measure of Adiposity in Identifying Cardiovascular Disease Risks among Thai Adults,” Obesity Research \& Clinical Practice, Vol. 2, No. 3, 2008, pp. 215-223. doi:10.1016/j.orcp.2008.05.003

[15] R. Taylor, I. Jones, S. Williams and A. Goulding, "Evaluation of Waist Circumference, Waist-to-Hip Ratio, and the Conicity Index as Screening Tools for High Trunk Fat Mass, as Measured by Dual-Energy X-Ray Absorptiometry, in Children Aged 3 - 19 Y," The American Journal of 
Clinical Nutrition, Vol. 72, 2000, pp. 490-495.

[16] A. Roche, "Growth, Maturation and Body Composition: The Fels Longitudinal Study 1929-1991,” Cambridge University Press, Cambridge, 1992. doi:10.1017/CBO9780511661655

[17] G. Lohman, A. Roche and R. Martorell, “Anthropometric Standardization Reference Manual," Human Kinetics,
Champaign, 1988.

[18] Y. Weili, B. He, H. Yao, J. Dai, J. Cui, D. Ge, Y. Zheng, L. Li, Y. Guo, K. Xiao, X. Fu and D. Ma, "Waist-toHeight Ratio Is an Accurate and Easier Index for Evaluating Obesity in Children and Adolescents," Obesity, Vol. 15, No. 3, 2007, pp. 478-752. 